\title{
NEW AGES FOR OLD CLUSTERS
}

\author{
I. N. REID \\ Palomar Observatory \\ 105-24, Caltech, Pasadena, CA 91125, USA
}

A major result of the Hipparcos mission is the availability of accurate parallaxes for a muchincreased sample of nearby subdwarfs. On the basis of those data, both Reid (1997-R97) and Gratton et al (1997-G97) have used main-sequence fitting techniques to obtain new estimates of globular cluster distances, deriving significantly larger moduli (by 0.15 to $0.3 \mathrm{mag}$ ) for extreme, metal-poor clusters such as M92 and M15. These re-calibrations are not solely the result of the revised parallax data - both D'Antona, Caloi \& Mazzitelli (1997 - DCM) and Reid show that similar results can be derived from pre-Hipparcos subdwarf data if the calibrations are not tied exclusively to HD 103095. The longer distance scale leads to a brighter inferred turnoff, and, combined with the latest models (DCM), ages of only 11 to 13 Gyrs - substantially younger than the $16 \pm 2$ Gyrs proposed by Bolte \& Hogan (1992).

Recently, Pont et al (1997) have suggested that these revised distances reflect systematic bias in the calibration, notably underestimation of subdwarf abundances. Their distance modulus for M92 $\left((\mathrm{m}-\mathrm{M})_{0}=14.68\right)$ is closer to the Bolte/Hogan value (14.65 mag) than those derived by G97 (14.82 $\mathrm{mag}$ ) or Reid (14.93 mag). While we believe that Pont et al overestimate the bias, particularly where high-resolution spectroscopic analyses are concerned (G97, Reid, preprint), there is no question that significant uncertainties remain in the cluster distance calibration. Colours, rather than luminosities, are particularly vulnerable, and a mismatch of 0.01 in $(\mathrm{B}-\mathrm{V})$ translates to $\delta(m-M)_{0} \sim 0.05$ mag. Amongst the problems are:

1) regardless of the random uncertainties in $[\mathrm{Fe} / \mathrm{H}]$ for subdwarfs, there are systematic differences of up to 0.3 dex between different calibrations (Reid, preprint);

2) the $\delta(B-V)$ correction required to adjust subdwarfs to a mono-metallicity scale is poorlydefined empirically;

3) interstellar reddenings remain uncertain for many subdwarfs and clusters;

4) discrepancies of up to 0.04 mag exist in the colours adopted for individual subdwarfs, and most stars have only BV photometry;

$5)$ there are systematic differences in the $\left(\mathrm{M}_{V},(\mathrm{~B}-\mathrm{V})\right)$ isochrones predicted by the DCM and Vandenberg models - in shape as well as zeropoint. These discrepancies are often ignored.

Overall, the main legacy of Hipparcos should be a better appreciation of the true level of uncertainty in main-sequence fitting. There are over 200 HIP stars with parallaxes measured to $\frac{\sigma_{\pi}}{\pi}<15 \%$, and falling below the 47 Tuc main-sequence. Accurate photometry and high-resolution spectroscopy of those stars will go a long way toward setting cluster distances on a firmer footing.

\section{References}

Bolte, M. \& Hogan, C. (1992) Conflict over the age of the Universe, Nature, 376, 399-402

D'Antona, F., Caloi, V. \& Mazzitelli, I. (1997) The Universe and globular clusters, $A p J, 477,519-534$

Gratton, R.G., Fusi Pecci, F., Carretta, E., Clementini, G., Corsi, C.E., \& Lattanzi, M. (1997), Ages of globular clusters from Hipparcos parallaxes of local subdwarfs $A p J$, in press

Pont, F., Mayor, M., Turon, C., Vandenberg, D.A. (1997) Hipparcos subdwarfs and globular cluster ages, Astr. Ap., in press

Reid, I.N. (1997) Younger and Brighter, $A J, 114$, pp. 161-179 